\title{
Acute and chronic effects of beetroot supplementation on blood pressure and arterial stiffness in humans
}

\author{
E. Turner ${ }^{1}$ and S. Mushtaq ${ }^{1}$ \\ ${ }^{1}$ Department of Clinical Sciences and Nutrition, University of Chester, UK
}

Dietary supplementation of beetroot juice, containing nitrate- a potent vasodilation agent, has been shown to be vasoprotective ${ }^{(1)}$, and dose dependent decreases in blood pressure (BP) have been previously demonstrated ${ }^{(2,3)}$. To our knowledge there has been only one study investigating the effect of beetroot supplementation in humans on arterial stiffness, measured using pulsewave velocity (PWV) and, although there was no effect of supplementation on PWV, there was a significant reduction due to beetroot supplementation in acute diastolic BP (3hrs, $P=0.023)^{(4)}$.

A double-blind, randomised, cross-over intervention trial was carried out in a cohort of 12 healthy male participants (mean age $\left.(\mathrm{SEM})=43(2 \cdot 1) \mathrm{yrs}, \mathrm{BMI}=27.8(1 \cdot 1) \mathrm{kg} \cdot \mathrm{m}^{2}\right)$ who underwent both beetroot juice and placebo supplementation for 14 days. The aim of the study was to assess the effect of $6.45 \mathrm{mmol}$ of nitrate in a concentrated $70 \mathrm{ml}$ beetroot drink (James White Ltd, Ipswich, UK) on systolic and diastolic BP, mean arterial pressure (MAP) and arterial stiffness (PWV, aortic augmentation index (Aix), brachial Aix) in humans.

BP and arterial stiffness measurements weretaken using PWV (Arteriograph, TensioMed,Hungary). Measurements were taken intriplicate at baseline, 3 hours post-supplementation (either beetroot juice orplacebo) and post-intervention (day 15). This was followed by a 7-day washoutperiod before participants were transferred to the alternate supplement.

Table 1. Effect of beetroot juice supplementation on BP and arterial stiffness.

\begin{tabular}{|c|c|c|c|c|c|c|c|}
\hline Parameter & Treatment & Baseline & SEM & $3 \mathrm{hrs}$ & SEM & Day 15 & SEM \\
\hline \multirow[t]{2}{*}{ Systolic BP (mmHg) } & Placebo $(n=12)$ & $127 \cdot 9$ & $2 \cdot 5$ & $129 \cdot 4$ & $3 \cdot 1$ & $129 \cdot 9$ & $3 \cdot 0$ \\
\hline & Beetroot $(n=12)$ & $130 \cdot 7$ & $2 \cdot 8$ & $127 \cdot 4 * *$ & $3 \cdot 2$ & $129 \cdot 5$ & $2 \cdot 9$ \\
\hline \multirow[t]{2}{*}{ Diastolic BP (mmHg) } & Placebo $(n=12)$ & $79 \cdot 8$ & 1.9 & $79 \cdot 1$ & $2 \cdot 2$ & $79 \cdot 7$ & $2 \cdot 2$ \\
\hline & Beetroot $(n=12)$ & 81.9 & $2 \cdot 8$ & $77 \cdot 2^{*}$ & $2 \cdot 5$ & $80 \cdot 3$ & $2 \cdot 5$ \\
\hline \multirow[t]{2}{*}{ MAP (mmHg) } & Placebo $(n=12)$ & $95 \cdot 8$ & $2 \cdot 1$ & $95 \cdot 9$ & $2 \cdot 3$ & $96 \cdot 2$ & $2 \cdot 4$ \\
\hline & Beetroot $(n=12)$ & $98 \cdot 1$ & $2 \cdot 7$ & $94 \cdot 0^{*}$ & $2 \cdot 6$ & $96 \cdot 8$ & $2 \cdot 5$ \\
\hline \multirow[t]{2}{*}{$\mathrm{PWV}(\mathrm{m} / \mathrm{s})$} & Placebo $(n=12)$ & $7 \cdot 9$ & $0 \cdot 3$ & $8 \cdot 0$ & $0 \cdot 4$ & 7.9 & 0.4 \\
\hline & Beetroot $(n=12)$ & $7 \cdot 9$ & $0 \cdot 3$ & $8 \cdot 0$ & $0 \cdot 3$ & 7.8 & $0 \cdot 7$ \\
\hline \multirow{2}{*}{ Aortic Aix (\%) } & Placebo $(n=12)$ & $15 \cdot 2$ & $2 \cdot 2$ & $14 \cdot 2$ & $2 \cdot 6$ & $14 \cdot 6$ & 1.8 \\
\hline & Beetroot $(n=12)$ & $15 \cdot 8$ & 1.7 & $12 \cdot 1^{*}$ & $2 \cdot 1$ & $14 \cdot 5$ & $1 \cdot 6$ \\
\hline \multirow[t]{2}{*}{ Brachial Aix (\%) } & Placebo $(n=12)$ & $-44 \cdot 9$ & $4 \cdot 1$ & $-46 \cdot 3$ & $5 \cdot 1$ & $-45 \cdot 4$ & $3 \cdot 6$ \\
\hline & Beetroot $(n=12)$ & $-43 \cdot 2$ & $3 \cdot 3$ & $-50 \cdot 5^{*}$ & $4 \cdot 2$ & $-45 \cdot 5$ & $3 \cdot 2$ \\
\hline
\end{tabular}

* Significant difference from baseline $(P<0 \cdot 05)$.

** Significant difference from baseline $(P<0 \cdot 01)$

Table 1 shows that there was no significant acute or short term effect of beetroot juice supplementation on the parameters measured when compared to placebo. However, there was a significant decrease in systolic $\mathrm{BP}(P=0.009)$, diastolic $\mathrm{BP}(P=0.035)$, MAP $(P=$ 0.017), aortic and brachial AIX ( $P=0.042$ and 0.041 respectively), 3hours post beetroot supplementation.

These results confirm previous findings ${ }^{(4)}$ that beetroot supplementation does not have an acute or short term effect on arterial stiffness measures. However, acute effects on arterial stiffness and BP within the beetroot juice supplementation group were observed. Further large scale studies on dietary nitrate supplementation and cardiovascular health are required to further assess efficacy.

1. Lundberg JO, Feelisch M, Björne H, Jansson EA, \& Weitzberg E (2006). Cardioprotective effects of vegetables: Is nitrate the answer? Nitric Oxide: Biology and Chemistry, 15(4), 359-362.

2. Kapil V, Alexandra BM, Okorie M, Maleki-Toyserkani S, Akram F, Farkhanda R, Arghandawi S, Pearl V, Benjamin N., Loukogeorgakis S, MacAllister R, Hobbs A, Webb A \& Ahluwalia A (2010). Inorganic Nitrate Supplementation Lowers Blood Pressure in Humans: Role for Nitrate-Derived NO. Hypertension, 56(2): 274-281

3. Webb A, Patel N, Loukogeorgakis S, Okorie M, Aboud Z, Misra S, Rashid R, Miall P, Deanfield J, Benjamin N, MacAllister R, Hobbs A \& Ahluwalia A (2008) Acute Blood Pressure Lowering, Vasoprotective, and Antiplatelet Properties of Dietary Nitrate via Bioconversion to Nitrite. Hypertension: 51(3): 784-790

4. Hobbs D, Goulding M, Nguyen A, Malaver T, Walker C, George T \& Lovegrove J (2013) Acute ingestion of beetroot bread increases endothelium-independent vasodilation and lowers diastolic blood pressure in Healthy Men: A randomized controlled Triall-4. J. Nutr. 143(9), 1399. 